Archives

$5 \mid 1990$

Varia

\title{
Les mages à la fin du Moyen Âge : un duo dynamique
}

\section{Richard C. Trexler}

\section{(2) OpenEdition}

\section{Journals}

\section{Édition électronique}

URL : http://journals.openedition.org/ccrh/2887

DOI : $10.4000 /$ ccrh.2887

ISSN : $1760-7906$

\section{Éditeur}

Centre de recherches historiques - EHESS

Édition imprimée

Date de publication : 15 avril 1990

ISSN : 0990-9141

Référence électronique

Richard C. Trexler, "Les mages à la fin du Moyen Âge: un duo dynamique », Les Cahiers du Centre de Recherches Historiques [En ligne], 5| 1990, mis en ligne le 20 mars 2009, consulté le 19 avril 2019. URL : http://journals.openedition.org/ccrh/2887 ; DOI : 10.4000/ccrh.2887

Ce document a été généré automatiquement le 19 avril 2019

Article L.111-1 du Code de la propriété intellectuelle. 


\title{
Les mages à la fin du Moyen Âge : un duo dynamique
}

\author{
Richard C. Trexler
}

1 Depuis quelques années, j'essaie de découvrir quelle est la signification des mages de l'Evangile dans la vie du Moyen Âge. Il m'a tout d'abord semblé que les mages - ou les Trois Rois, autre nom donné dans l'Occident médiéval à ceux qui, d'après Matthieu, sont venus reconnaître l'Enfant Roi Jésus - représentent l'unique autorité vraiment universelle dans le Nouveau testament. D'autres constatations ont suivi. Ainsi, Franz Cumont a montré, il y a longtemps, que les premières représentations sculptées de l'Adoration des mages étaient des transcriptions chrétiennes de représentations plastiques du triomphe impérial romain ${ }^{1}$. Il devenait donc clair que dans la liturgie et dans les arts, comme dans les spectacles de rues du Moyen Âge, les mages, en marche vers l'Adoration, constituaient l'image par excellence utilisée pour représenter le triomphe de la Chrétienté, mais aussi bien la victoire de royaumes et de cités, et celle de nombreux individus de sexe masculin ayant exercé une domination.

2 Ainsi, le thème des mages évoquait de façon générale le pouvoir et le contrôle. Il semblerait, par exemple, que les mages n'aient fait leur entrée dans l'art chrétien que lorsque les évêques eurent reconu Constantin pour leur souverain, au début du IV siècle ; de fait, le Signe que Constantin vit dans le ciel pourrait être assimilé à l'Étoile de Bethléem ${ }^{2}$. On retrouve ultérieurement, au cours des âges, cette association symbolique avec le pouvoir: ainsi, dans les mosaïques de Saint Vital de Ravenne, Justinien et Théodora offrent des présents à Jesus; le premier est représenté avec «l'Étoile » de Constantin à son côté ; Théodora porte le présent dans les mains et sa robe est décorée d'une figuration de l'Adoration des mages ${ }^{3}$. Pendant le haut Moyen Âge, Nicolas de Bari faisait la louange de son seigneur Frédéric II en tant que petit-fils de Frédéric Barberousse et fils de l'empereur Henri IV, tous deux décédés. «Ces trois empereurs », disait Nicolas, «sont comme les trois mages, qui sont venus avec des présents adorer dieu fait homme ... et lui est le plus jeune (adolescentior) des trois, sur qui l'Enfant Jésus a posé ses mains bienheureuses et ses bras sacrés $»^{4}$. Puis, à la Renaissance, des artistes ont figuré sous les 
traits des Trois Rois, des membres de la famille des Habsbourgs et d'autres dynasties, tandis que des explorateurs partaient à la recherche « du (des) pays d'où était venu le roi qui avait apporté de l'or à Jésus »; après les avoir conquis, ils mirent en scène des processions de rois indigènes, en route vers la crèche de l'Epiphanie, comme pour soumettre de nouvelles nations à la domination de Madrid et de Rome ${ }^{5}$. Dans l'Ancien Monde, le comportement de ces trois monarques devant l'Enfant Jésus était un modèle de prière et de dévotion ${ }^{6}$; de même, les habitants du Nouveau Monde pourraient apprendre de ces monarques la leçon de leur propre soumission.

Hélas, depuis le troisième siècle, la tradition a minimisé le rôle des considérations politiques qui ont présidé à la diffusion de cette histoire des mages, au profit d'interrogations historiques concernant, entre autres, le nombre et l'origine des mages, questions auxquelles, du fait du silence de l'évangéliste, il était impossible de répondre. Néanmoins, on en vint généralement à supposer, au Moyen Âge, que les mages venaient de trois contrées différentes, et même de continents différents à l'intérieur de «l'Orient » dont parle Matthieu, plutôt que du même lieu? ${ }^{7}$. Cependant, comme nous le verrons, il y avait des variantes concernant cette origine des mages ; elles dépendaient, probablement, en fait, des préoccupations politiques de ceux qui utilisaient cette histoire à des fins politiques.

4 Le nombre des mages constituait apparemment un problème bien plus simple et, de ce fait, susceptible de moins de variations. Bien qu'on puisse trouver, dans l'art chrétien primitif, deux, trois, quatre ou même douze mages, le consensus quasi universel des savants et des artistes était qu'il y avait eu trois mages apportant chacun l'un des présents cités par l'évangéliste, ce trio symbolisant, de toute évidence, la trinité, les trois âges de l'homme, etc. Cependant, tandis que les mages sont toujours représentés à trois durant le Moyen Âge et la Renaissance, leur représentation visuelle laisse souvent entendre qu'ils ne sont que deux. Je me propose ici de mettre en lumière la persistance d'une certaine dualité du groupe des mages dans les textes médiévaux. Je traiterai ces questions de lieu et de nombre en me référant particulièrement à la tradition dramatique de la fin du Moyen Âge, à commencer par celle de Florence, en Italie.

Il est maintenant certain, si l'on s'en tient aux traditions politiques établies, que la famille des Visconti de Milan a utilisé le culte des mages lors de son élévation au statut ducal (1395), et que, peu après, la famille des Médicis dans la Florence républicaine, a utilisé le même culte dans sa recherche de l'autorité princière ${ }^{8}$. L'expression institutionnelle de l'attachement de cette famille aux Rois était la Compagnie ou confrérie des mages qui, en tant qu'instrument des Médicis, comptait parmi ses membres une part significative de l'élite florentine du Quattrocento. Son influence était si grande qu'on en parlait comme de «la république des mages », et qu'on la considérait comme l'une des deux confréries de la cité qui garantissait la sécurité politique à ses membres.

6 S'appuyant sur des traditions antérieures, ce groupe a facilité le développement d'une géographie « royale » dans la Florence républicaine : un certain nombre de « roitelets » de quartier se rangeaient sous l'autorité des Trois Rois ou de la Compagnie des mages, qui elle-même ne rendait des comptes qu'aux Médicis. Puisqu'il s'agissait de royaumes de fiction, la république pouvait tolérer l'opposition inhérente à ses propres fêtes et à sa propre géographie que constituait cette structure monarchique. La manifestation publique des mages était suffisamment évidente lors de l'Epiphanie ou fête des mages (5-6 janvier) et pendant la fête civique de saint Jean-Baptiste (20-24 juin), fêtes au cours desquelles les Trois Rois se rendaient en grandes cavalcades auprès de la crèche dans 
l'église de San Marco, certainement accompaniés par les petits rois qui se trouvaient sous leur autorité et qui représentaient chacun un quartier de Florence ${ }^{10}$

7 Les sources qui permettraient de retracer l'histoire interne de ce "gouvernement parallèle» $\mathrm{au} \mathrm{xv}^{\mathrm{e}}$ siècle restent encore à trouver. Le plus important, dans la documentation fragmentaire qui a été retrouvée et qui a été incorporée a la littérature concernant le sujet, a cependant des implications assez significatives. Il s'agit d'une lettre qui a pour la première fois été analysée par Rab Hatfield, et dans laquelle Caspar, Melchior et Balthasar s'excusent auprès de la confrérie de San Bartolomeo de ne pouvoir participer à ses prochaines festivités : cela leur était impossible, disaient-ils, parce qu'ils étaient occupés à "transformare i nostri regni - et maximamente Egiptto, Ethiopa e Nubia, Arabia, Sabea, India, Madia e ll'una e ll'altra, e'rmenia $»^{11}$. Ainsi, trois royaumes africains et cinq royaumes asiatiques, sans compter l'Arménie, soit neuf quartiers ou plus, étaient gouvernés et en cours de réorganisation par les trois rois. Remarquons que la ponctuation «e » semble isoler les uns des autres les trois premiers royaumes, les cinq suivants, et finalement l'Arménie. Le royaume d'origine de chacun des mages n'apparait pas clairement, mais cela ne doit pas nous empêcher de faire l'hypothèse que l'Arménie ait dû être l'un d'entre eux, non seulement en raison de sa séparation syntaxique dans le texte, mais en raison de la tradition à la fois chrétienne et asiatique de l'Arménie. Toutefois, la présence de l'Arménie parmi ces royaumes est en elle-même surprenante, puisqu'il n'existe pas de tradition établie qui revendique l'Arménie comme patrie de l'un des trois mages ${ }^{12}$.

8 Cela est vrai dans tous les textes à l'exception d'un seul, dont l'importance a jusqu'à présent été négligée. Ce texte permet précisément de démontrer à quel point les noms des patries des rois trouvaient un écho dans les conditions locales. Je fais référence à une sacra rappresentazione della festa de' Magi dont ses éditeurs disent que, d'un point de vue linguistique, elle n'est pas d'origine florentine. Néanmoins, le texte a certainement été " florentinisé »" ${ }^{13}$. On peut l'affirmer surtout en raison de l'origine attribuée à l'un des rois. Dans cette pièce, le premier roi, celui qui porte l'or (Balthasar) est le souverain couronné de l'Arabie, tandis que le troisième, celui qui porte la myrrhe (Caspar), est le seigneur de l'Inde Supérieure - deux contrées traditionnellement considérées comme des patries des mages. Melchior, le second roi, celui qui porte l'encens, règne sur la Grande Arménie et est connu chez les peuples d'Orient ${ }^{14}$. Voilà donc ici un mage d'Arménie, et nous savons que Florence comptait un royaume fictif du même nom.

9 La raison pour laquelle un royaume florentin fictif portait le nom d'Arménie est que Florence comptait un quartier qui avait quelque chose à voir avec des Arméniens; les autres royaumes avaient également de telles connotations. Ainsi l'Arabie, connue pour la couleur rouge de son sol, faisait probablement référence à la Città Rossa, royaume situé dans la paroisse de San Ambrogio; et l'on peut présumer que l'Inde s'identifiait à la paroisse de San Tommaso, dont l'église qui se trouvait sous le patronage des Médicis, faisait face, sur le Mercato Vecchio, à l'inscription India tibi cessit. Tirant sa dénomination de l'existence d'un monastère de fères arméniens, "Armenia " était également un quartier connu par ailleurs comme le Canto alle Macina ou royaume de La Meule. Proche du palais des Médicis et de son église paroissiale de San Lorenzo, ce quartier s'était acquis une réputation de loyauté à la famille des Médicis ${ }^{15}$.

10 L'identité festive de ce quartier remontait au moins à 1439 et au Concile de Florence, car à cette occasion le groupe du Canto mit en scène le jeu de la Résurrection, qui fit sa réputation. On peut supposer que le faux sépulcre ou munimento censé contenir le corps 
de Jésus, ainsi que d'autres objets utilisés pour les festivités, étaient déjà conservés par cette confrérie ${ }^{16}$. Dans ce contexte, une lettre récemment découverte par William Kent prend toute son importance. Le 13 juin 1439, tandis que l'Empereur byzantin se trouvait au Concile qui préparait l'union de l'Eglise grecque et de Rome (6 juillet), Lorenzo di Giovanni de Medici, depuis la campagne où il se trouvait, donna l'ordre au secrétaire de son frère Cosimo à Florence, de fournir au porteur de la lettre, simple ouvrier agricole (lavoratore), un manteau d'allure grecque. Cet homme devait ensuite se rendre auprès de l'Empereur comme émissaire du Roi d'Arménie ${ }^{17}$.

11 Du fait qu'il n'existait pas de véritable « Roi d'Arménie » - le pape réalisa l'union avec de simples « ambassadeurs des Arméniens »- Kent a probablement raison de penser que ce Roi d'Arménie était en réalité un monarque des festivités florentines; bien plus, le Roi d'Arménie pourrait n'avoir été autre que Lorenzo lui même, puisque c'était lui qui avait envoyé cet ouvrier en ambassade. Par ailleurs, l'idée de Kent selon laquelle l'Empereur qui reçut cette ambassade était le monarque du quartier de la Città Rossa, est moins évidente. Il était en fait d'usage à cette époque que les royaumes de théâtre entretiennent des rapports avec de véritables monarques, de telle sorte que l'Empereur en question peut très bien avoir été le véritable Empereur, l'ambassade étant alors pour les Médicis un moyen très clair de faire savoir qu'ils soutenaient les revendications byzantines sur l'Arménie, qui se trouvait alors sous le contrôle des Tartares.

Quoiqu'il en fût, ce qu'il faut souligner c'est qu'une cité pouvait dans le même temps mettre en forme une géographie urbaine ludique et modifier les origines traditionnellement attribuées aux mages pour faire place à une localisation urbaine existante de caractère non-ludique, telle que l'Arménie. A son tour, la géographie des mages pouvait faire de nombreux quartiers autant de petits royaumes placés sous l'autorité des mages. Finalement, autant qu'on puisse le dire, les trois royaumes des mages ou sa Compagnie témoignaient effectivement de l'existence d'une force à la fois festive et politique, celle des Médicis.

13 J'en viens maintenant à la question du nombre des mages. Si le nombre et le nom des royaumes placés sous l'autorité unique de la Compagnie des Mages pouvaient être manipulés pour refléter les véritables identités de quartiers des cités, il pourrait en être de même pour le «nombre perçu » des mages, si je peux me permettre cette expression. En effet, il nous faut tout d'abord reconnaître qu'un certain dualisme a toujours soustendu la triade physique des mages. Le premier type de dualisme oppose l'unité des trois mages à une autre unité extérieure : j'ai déjà mentionné le duo vivant composé de Justinien et Théodora qui, tel qu'on le voit à Ravenne, dominait et même s'opposait non seulement à l'Enfant Jésus, mais également aux mages morts. Les sculptures qui figurent sur certains sarcophages contemporains de San Vitale sont peut-être particulièrement caractéristiques de ce dualisme intérieur-extérieur tel qu'il s'exprime dans l'art. Le motif est pratiquement invariable: les trois mages qui apportent des présents à Marie et à l'enfant ne constituent en réalité qu'une seule forme. Comme dans la plupart des premières Adorations chrétiennes, ils s'avancent d'un seul pas et sont tous vêtus de la même façon - comme tant d'ambassadeurs qui représentent un seul roi ; contrairement à ce qui se faisait en Occident, les Byzantins n'ont jamais représenté les rois mages couronnés. Derrière ce groupe, s'avance une figure qu'on peut appeler aujourd'hui le " quatrième mage ». Cependant, comme ces premiers mages chrétiens sont identiques, il apparait comme un « second » mage qui ferme la marche, apportant son propre présent, 
comme pour affirmer qu'il avait, lui aussi, au cours de sa vie, offert des présents à Dieu et à l'église, et que, de ce fait, il méritait maintenant le ciel ${ }^{18}$.

Un second type de dualisme sépare les mages eux-mêmes en deux entités, phénomène qui n'est apparu que lorsque les mages ont cessé de marcher d'un seul et même pas, et ont commencé à assumer une certaine individualité. J'ai déjà fait remarquer un certain type de dualisme dans la louange à Frédéric II, qui date du XIII ${ }^{\mathrm{e}}$ siècle ; on le glorifie beaucoup plus que ses prédécesseurs, bien plus, on l'oppose à eux. Ainsi, tout en préservant la vision formelle de la trilogie constituée de Balthasar, Caspar et Melchior, les hommes de la fin du Moyen Âge ont commencé à attribuer, de façon imaginative et figurative, une polarité interne à ce groupe qui apporte des présents. Je souhaite démontrer maintenant à quel point il était tout à fait possible de percevoir cette figuration visuelle de trois personages comme un duo.

À la fin du Moyen Âge, on suggérait aux spectateurs de certaines représentations de rues mettant en scène les mages, que le premier et le second roi étaient identiques sous un rapport essentiel, qui les distinguait fortement du troisième roi. Ainsi, en ce qui concerne leur rapport à l'Enfant et, contrairement à notre interprétation habituelle, deux entités et non trois apportaient des présents. C'est Norbert King qui, le premier, a mis en évidence ce schéma littéro-dramatico-visionnaire. Il mérite qu'on s'y arrête et qu'on l'analyse de plus près. Cette différence essentielle était une différence d'âge ; il existe quatre types d'histoires qui expriment le comportement des rois jeunes et des rois âgés, en ce qui concerne les problèmes de préséance et de dévotion. Dans le premier type, composé de jeux non-liturgiques originaires de France et de Suisse, une discussion s'élève parmi les mages pour savoir qui ira le premier adorer l'enfant. Au terme de ce débat, c'est le roi le plus âgé qui reçoit la préséance tandis que le plus jeune reste à l'arrière, comme le decorum pouvait le laisser supposer ${ }^{19}$.

L'inattendu se produit dans le second type d'histoire, qui commence avec un jeu nonliturgique du début $d u \mathrm{xv}^{\mathrm{e}}$ siècle, originaire d'Erlau. Ici, l'empressement du troisième roi à être le premier à offrir son présent à l'enfant est tel que le roi le plus âgé cède, permettant au plus jeune de passer le premier, et lui donne son masque de vieillard, que le jeune roi ainsi vieilli va revêtir. Naturellement, le roi le plus jeune assume les vertus de l'âge, de même que le plus âgé, rajeuni, retrouve la jeunesse et ses privilèges ${ }^{20}$. King a certainement raison de souligner le caractère miraculeux de cette histoire, mais c'est surtout le message qui importe. Sans qu'il soit question d'un âge intermédiaire, ce message est le suivant : si vous voulez bénéficier de la préséance, vous devez être prêt à renoncer à l'eros de la jeunesse, et vice versa. La même histoire, pratiquement, apparaît dans un manuscrit contemporain de Brixen : le jeune roi est prêt à renoncer à la jeunesse pour être le premier à approcher l'enfant. La divinité en a fait le plus âgé des trois, sur quoi les deux autres rois lui ont cédé le pas ${ }^{21}$. Dans ce cas, le second roi était au début probablement plus âgé que le troisième, mais, encore une fois, il ne joue absolument aucun rôle dans l'opposition entre sénilité et jeunesse, qui est mise en scène.

Une troisième histoire révèle toutefois l'âge de ce roi intermédiaire, et, comme on le verra, elle conforte cette idée d'une dualité du goupe des mages. Le lieu de création de cette histoire ne se trouvait pas loin de Brixen, dans le couvent de femmes de la petite ville lombarde de Binasco, à mi-chemin entre Milan et Pavie, cités réputées toutes deux pour leurs festivals et leurs monuments consacrés aux mages ${ }^{22}$. Dans la vision de Véronique de Binasco, qui date des années 1490, les trois mages discutaient pour savoir qui irait le premier adorer l'enfant. Au terme de ce débat, dit Véronique, c'est le plus 
jeune des trois qui s'approcha le premier de l'enfant et lui baisa les pieds. Sans préciser l'ordre dans lequel ils se succédèrent, si toutefois il y en avait un, notre source dit qu'alors «les autres firent de même $»^{23}$. C'est à ce moment que Véronique vit que «l'on s'aperçut avec saisissement que le plus jeune roi, qui avait adoré le premier, - notons ici les termes employés - était devenu plus âgé et avait maintenant le même âge que les deux autres $»^{24}$. Ainsi, l'auteur, tout à fait à l'encontre des idées reçues, affirmait que les deux rois les plus âgés étaient du même âge. L'axe central de toute cette histoire était la tension entre " âge » et "jeunesse », tension qui fut résolue en éliminant la différence. Mais jusqu'alors, il y avait effectivement deux et non pas trois types de mages.

Il est possible que Véronique ait pensé que le vieillissement du jeune roi était une punition; il se pourrait donc que notre quatrième et dernier document ait une teneur morale comparable à celui-ci et différente de celle des récits antérieurs. Dans son Degli errori dei pittori (1563), Giovanandrea Gilio da Fabriano fait référence à une croyance répandue à l'époque, quand il insiste pour que les peintres s'astreignent à représenter les personnages à l'âge qui leur est attribué dans la Bible: le jeune ne doit pas être représenté âgé, comme l'était le premier mage, à ce qu'on dit généralement. Il venait pour offrir l'or, la myrrhe et l'encens à notre Seigneur. Etant jeune, et refusant néanmoins de céder son droit de préséance au plus âgé qui aurait offert ses présents avant lui, il devint vieux, de façon miraculeuse, et le plus âgé devint jeune ${ }^{25}$.

Ainsi, dans cette version, il apparaît que le jeune homme a été puni pour avoir usurpé l'honneur de la préséance, et le vieil homme recompensé pour avoir souffert l'indignité. Une fois encore, il n'est pas fait mention du second roi ; pour ce conte moral, il n'existe effectivement que deux rois, une fois encore. Cette fois-ci cependant, notre source semble dire que, lorsqu'ils regardaient la figuration des mages dans les représentations artistiques, certains contemporains n'étaient pas abusés par les apparences picturales, et savaient que le premier roi, apparemment âgé, était en réalité jeune, et que le jeune roi était en réalité plus âgé. L'auteur suggère en fait que les spectateurs percevaient deux rois, opposés l'un à l'autre. Le lien entre perception et art nous ouvre un aperçu précieux des sources visuelles possibles de cette polarisation des mages, sur laquelle je vais revenir brièvement.

20 J'ai montré que la tendance à instaurer une polarité interne des mages n'avait rien de neuf à la fin $\mathrm{du} \mathrm{Xv}^{\mathrm{e}}$ siècle: dès que les mages ont cessé d'être représentés comme identiques l'un à l'autre, il est manifeste, par exemple, que le troisième roi vivant a été opposé aux deux premiers rois morts, ou que, le troisième roi pauvre a été opposé aux deux premiers rois riches ${ }^{26}$; on pourrait multiplier les types de polarisation interne. En fait, ce qui pourrait être nouveau à cette époque, c'est l'insistance sur la préséance comme procédant de l'âge. Dans ce conflit, le second roi, a le même âge que le plus âgé, ou bien alors n'existe tout simplement pas.

21 Mon propos ici n'est pas d'essayer d'expliquer l'émergence du motif du conflit des générations dans l'histoire des mages. Ce motif est peut-être en relation avec l'importante augmentation de la population en Italie à la fin $d u \mathrm{xv}^{\mathrm{e}}$ siècle; il éclaire certainement les possibilités économiques et politiques qui s'offraient ou se dérobaient à la jeunesse. De toute évidence, l'accent mis sur le conflit des générations à cette époque ne se limite pas à cette seule histoire. Dans l'Europe du $\mathrm{xv}^{\mathrm{e}}$ siècle, on portait, sous bien des aspects, une grande attention à la jeunesse, ce qui était un fait nouveau, particulièrement à Florence, où des modifications institutionnelles importantes se cristallisaient autour de ces tensions entre générations ${ }^{27}$. Sans aucun doute, il nous faut 
garder à l'esprit que les conflits d'âge entre les mages avaient pour enjeu la première place, juste au dessous d'une divinité qui disposait d'une autorité incontestable; on n'était pas pour rien à la veille de l'âge du principat. Cependant, la bataille des mages permet de faire une petite avancée de plus dans une recherche sérieuse concernant les relations entre générations à cette époque.

Il semblerait, en fait, que ce thème des miracles de l'âge soit beaucoup plus ancien que nos sources actuelles ne le laissent supposer, et qu'il procède de jeux liturgiques plus anciens donnés dans les églises. Dans ce cadre, ceux qui jouaient les rois étaient souvent des clercs - ce qu'on appellerait aujourd'hui des enfants de choeur - du même âge. C'est pourquoi, on peut faire l'hypothèse que pour assumer des âges différents sur la scène liturgique, les deux premiers rois mettaient des barbes ou des masques, tels qu'on les voit représentés dans l'art. J'ai fait allusion plus haut à un masque de ce type utilisé dans un jeu non-liturgique, et on peut retrouver dans au moins une illustration antérieure, des masques portés par les jeunes ${ }^{28}$. Conformément à cette notion, l'histoire du «miracle de l'âge ", qui est elle-même fonction de la réalité sociale et politique de l'époque, a été construite à partir d'une pratique théâtrale. Notons qu'à Florence, au Xve siècle, dans une autre circonstance de profonde polarisation entre l'âge et la jeunesse - à savoir les festivals - des groupes de fils s'avançaient masqués en imitant à la perfection leurs pères, qui en étaient abasourdis ${ }^{29}$.

Ne négligeons pas cependant la forêt de la structure au profit des arbres de la chronologie. La tendance persistante à un dualisme sous-jacent dans les représentations des trois rois qui vinrent adorer l'enfant Jésus témoigne peut-être d'un besoin plus profond de polarisation des entités qui, comme les mages, revendiquent d'être des représentations totalisantes de subordination ou de pouvoir. Disons les choses autrement: le trio des mages devait inclure un élément qui reste, pour ainsi dire, en dehors du cercle de la respectabilité. La fonction des mages, chez Matthieu, n'était-elle pas de faire reconnaitre Jésus par des étrangers, des gens de l'extérieur, venant de contrées lointaines, qui proclameraient roi l'enfant de l'intérieur? Dans l'univers chrétien post-constantinien, où les mages sont arrivés pour être honorés comme des figures du culte intérieur, l'étranger persiste dans son rôle.

Cette esquisse n'est fondée que sur des sources écrites. Cependant, même en l'absence de représentations picturales, le lecteur s'est certainement aperçu que je n'avais pas mentionné le principal étranger qui figure dans les Voyages de l'Adoration, dans l'art européen de la fin du Moyen Âge : le noir, troisième roi mage; noir qui est toujours représenté comme une figure exotique, et souvent efféminée. Les artistes ont souvent évoqué l'effemination des noirs en les représentants avec des anneaux aux oreilles; je montrerai ultérieurement qu'ils apprenaient aussi à représenter un troisième roi blanc efféminé, par d'autres moyens.

On peut dire, à la limite, qu'une intrusion importante du genre opposé, le genre féminin, était à l'oeuvre dans l'histoire des représentations des mages. Il faudrait une autre étude qui se focaliserait sur des preuves visuelles pour le démontrer. Tenons-nous en à ceci : des hommes comme Matthieu se sont tournés vers l'Orient en quête de mages extérieurs qui prouveraient que leur Seigneur était roi et dieu. Alors même que les Trois Rois venaient administrer la preuve d'un consensus solennel d'hommes âgés, une vitalité qui n'avait rien de solennel, faisait intrusion : le danger de la jeunesse, la menace de la femme, la séduction du noir. Si les Trois Rois exprimaient l'obéissance d'hommes politiques, ce duo 
dynamique offrait plus largement ses présents pour le compte de la société et de la nature au sens large.

\section{NOTES}

1. F. CUMONT, "L'Adoration des mages et l'art triomphal de Rome " in Memorie della pontifica accademia romana di archeologia, 3 (1922-23), pp. 81-105. Mes contributions antérieures à la problématique des mages sont les suivantes: Church and community, 1200-1600. Studies in the history of Florence and New Spain, Rome. 1987. ch. 3, 4, 10 Traüme der heiligen Drei Könige » in Iconographie der Traüme im Mittelalter, eds. A. PARAVICINI-BAGLIANI et G. STABILE, Stuttgart, 1989 ; et "Triumph and Mourning in North italian Magi Art », à paraître dans Art and Politics in Late Medieval and Early Renaissance Italy : 1250-1500, ed. C. ROSENBERG. South Bend. 1990.

2. J. DECKERS, «Die Huldigung der Magier in der Kunst der Spätantike », in Die heiligen Drei Könige. Darstellung und Verehung. Katalog zur Ausstellung des Wallraf Richartz Museums in der Josef-Haubrich-Kunsthalle Köln, I, Dez. 1982 bis 30 Jan. 1983, ed. Rainer BUDDE, Cologne, 1982, pp. 20-32. Un des premiers exemples du labarum considéré comme l'Étoile se trouve reproduit dans G. Wilpert, Ein Cyclus christologischer Gemälde aus der Katakombe der heiligen Petrus und Marcellinus, Freiburg/B, 1891.

3. Les caractéristiques des mages de Saint Vital sont brillamment analysées dans O. von SIMSON, Sacred fortress. Byzantine art and statecraft in Ravenna, Chicago, 1948.

4. «Profecto hii tres imperatores sunt quasi tres magi, qui venerunt cum numeribus deum et hominem adorare. sed hic est adolescentior illis tribus, super quem Ihesus felices manus posuit et brachiola sacrosancta». R. KLOOS, "Nikolaus von Bari, eine neue Quelle zur Entwicklung der Kaiseridee unter Friedrich II » in Deutsches Archiv für Erforschung des Mittelalters, 11, (1954-1955), 171 (1235).

5. Le langage de Colomb se trouve dans « La vie ludique dans la Nouvelle Espagne. L'Empereur et ses Trois Rois ", dans mon livre Church and community, pp. 493-510 ; voir aussi les passages très éclairés concernant les mages et les explorations dans Jean DEVISSE et Michel MOLLAT, Africans in the christian ordinance of the world (fourteenth to the sixteenth century), vol. 2, 2e partie de The image of the black in western art, New York, 1979.

6. Voir BUTTNER, Imitatio, pp. 19-33.

7. Le texte classique qui renvoie à ce type de question est, bien sûr, la Légende des Rois de Johannes d'Hildesheim, écrit vers 1375 : une synthèse récente en a été faite et se trouve dans P. KAPLAN, The Rise of the black magus in western art, Ann Arbor, 1985, pp. 19-70.

8. L'étude fondamentale sur cette question a été faite par R. HATFIELD, "The Compagnia de'magi », in Journal of the Warburg and Courtauld Institutes, 33 (1970), pp. 107-161. Pour une plus étude plus récente, voir mon livre Triumph, cité plus haut. Voir aussi E. MERLINI, « Il trittico eburneo della Certosa di Pavia: iconografia e committenza ", in Arte Cristiana, 73 (1985), pp. 369-383 ; 74 (1986), pp. 139-154.

9. Vers 1470, il Zampillo était l'autre nom que l'on donnait aux mages : « Compagnia del Zapillo, cioè, de' Magi che si rachuna in San Marcho "; Archivio di Stato, Firenze, Giorname del massaia delle camera della arme, $\mathrm{n}^{\circ} 7$ (1494-1495), fo 9r (24 mar. 1494 st. com.). Ainsi : "Chi è della (compagnia della) Chrocietta e del Zampillo puo dire lo stato suo esser tranquillo »; Dei, cité dans M. PISANI, Un avventuriero del Quattrocento, Genova, 1923, p. 105. Voir la lettre en vers de Francesco Alberti, 
membre de la confrérie, qui s'adressait en termes identiques au plus haut officier du gouvernement de Florence, le gonfalonier de Justice, pour obtenir certains crédits qui lui étaient dus : A. LANZA, Lirici toscani del'400, Rome, 1973, 122f $\mathrm{f}^{\circ}$.

10. R. HATFIELD, «Compagnia »; R. TREXLER, Public life in Renaissance Florence, New York, 1980, pp. 401-459.

11. R. HATFIELD. «Compagnia ». p. 148. J'ai daté cette lettre de 1471 ; Public life. p. 401.

12. Il existe une seule exception : c'est l'histoire qui raconte que Caspar, bien que non originaire d'Arménie, y a été enterré ; H. KEHRER. Die heiligen Drei Könige in Literatur und Kunst, I. Leipzig. 1908. p. 82. Johannes d'Hildesheim dit que les mages se sont arrêtés en Arménie alors qu'ils faisaient route vers Bethléem : le texte se trouve dans C. HORSTMANN, The Three Kings of Cologne. London. 1886, p. 281.

13. N. NEWBIGIN (ed.), Nuovo corpus di sacre rappresentazioni florentine del Quattrocento, Bologna, 1983, pp. 183-203. L'auteur y reprend la thèse de Bartholomaeis et dénie formellement au texte une origine florentine. Cependant, dans une correspondance privée. Newbigin reconnaît que l'original comporte « une forte patine florentine ». Elle pense que la langue d'origine est du Nord de l'Italie, ce qui est une conclusion intéressante lorsqu'on connaît l'arrière-plan milanais du culte florentin dont il a été question (cf. note 8).

14. NEWBIGIN, Nuovo corpus. pp. 185. 193. 201.

15. Les garzoni e giovani du Canto alle Macina vinrent rapidement en aide à Lorenzo de Medici lors de la conspiration des Pazzi en 1478 : D.V. et F.W. KENT. « Two vignettes of florentine society in the fifteenth century ». in Rinascimento, ser. 2. 23. 1983. pp. 252-260.

16. R. TREXLER, Public life. pp. 402-408.

17. «Voi. ser Alesso, acchatate una cioppa alla ghrecha a Bechone e mandatelo per imbasciadore allo 'Mperadore per parte del re d'Armenia, et ghua(r)date s'elli à viso da ccio »; F.W. KENT et P. SIMONS, Patronage, art and society in Renaissance Italy, Oxford, 1987. p. 10. Ceci est particulièrement intéressant parce que l'union entre Rome et l'Eglise arménienne se produisit le 22 novembre, soit trois mois après le départ de l'Empereur, qui eut lieu le 16 août; G. CAMBI, « Istorie », in Delizie degli cruditi toscani, XX, Florence. 1785, pp. 218, 220, 225 ; L. MORELLI, «Cronaca ». in Delizie, Florence, 1784, p. 170 : L. von PASTOR, Geschichte der Päpste im Zciltalter der Renaissance, I, Freiburg, 1955, p. 331.

18. L'une des sources de cette polarisation des mages porteurs de présents se trouve certainement dans le conte d'Aelian (c. 170-c. 230 A.D.), qui parle d'un homme qui a été enlevé et qui offre de l'eau à un Artaxerxès plein de reconnaissance; celui-ci lui dit que son présent est aussi précieux qu'aucun autre présent qu'il ait reçu: Varia Historia, Leipzig, 1974, pp. 12ff (bk I, ch. 31-32). Cf. William PAINTER, The palace of pleasure (1566), London. 1890, pp. 54f (roman 9); et C. MANSON, A gift for the king. A persian tale, retold and illustrated by CM. New York, 1989. En ce qui concerne l'acception moderne de cette notion, voir le roman de Michel TOURNIER, Gaspar, Melchior, Balthazar, traduit en anglais sous le titre The four wise men, New York, 1984.

19. En ce qui concerne les jeux de ce type, voir N. KING, Mittelälterliche Dreikönigsspiele, Freiburg/ S, 1979, p. 203.

20. Ibid., pp. 130-131.

21. Ibid.

22. Voir ma contribution « Triumph and mourning ", à paraître.

23. «Anteaquam reges infantulum contingentes adorarent ter genuflexere, diuque ad invicem disputarunt, quis eorum prior adoraret: denique junior primus accessit, pedes infantuli solo exosculans, et quam capite gestabat coronam ante pedes infantuli reponens. quem Iesus infans benedixit. Sie quoque egere ceteri ». La vision se produisait au cours de périodes successives d'extase, qui commençaient en privé et se poursuivaient à différents moments lors des messes qui suivaient. La vita. par Isidoro Isolani(ca. 1518) se trouve dans Acta Sanctorum, Jan. (1) 13, ch.IV, p. 906. 
24. "At reges specie corporis praestantes fuisse dicebat Veronica, aureis vestibus usque ad genua indutos, iunioremque regem, qui primus adoraverat. veluti effectum seniorem et unius actatis qui reliquis duobus demirans intuita est ». Ibid.

25. «Né vecchio giovine, come il vulgo dice del primo Re Mago che ando a offerire l'oro, la mirra e l'incenso al signor nostro, che, essendo egli giovine né volendo cedere ad un vecchio che prima di lui offerisse, egli ne divenne miracolosamente vecchio, et il vecchio giovine ». Giovanandrea GILIO da FABRIANO, « Degli errori de'pittori circa l'istorie », in Trattati d'arte del cinquecento II, Bari, 1961, p. 45.

26. Notons que les trois ordres classiques de la société reposaient aussi de fait sur une dualité entre riches et pauvres; ainsi, dans la tradition littéraire, il pouvait se faire que le troisième mage soit pauvre et les deux premiers riches; voir mon texte Church and community, pp. 507-508. Dans une variante des sacre rappresentazione des mages à Florence, décrites plus haut, le second roi Melchior dit : «Il mio è piccol tesoro; cioè incenso... ». NEWBIGIN, p. 201.

27. Voir mon article «Ritual in Florence : adolescence and salvation in the Renaissance ", in The pursuit of holiness in late medieval and Renaissance religion, ed. C. TRINKAUS et H. OBERMAN, Leiden, 1974, pp. 200-264.

28. Voir cette illustration originaire de Berlin dans W. COOK, "The earliest painted panels of Catalonia (VI)», in Art Bulletin, 10, 1928, p. 320 (fig. 16). Si les peintures représentent parfois ces jeux, on devrait pouvoir trouver d'autres rois âgés dans les peintures médiévales, représentés avec une légère ligne verticale sur le visage, qui passe devant les oreilles, et indique l'existence d'un masque.

29. R. HATFIELD, « Compagnia », p. 116.

\section{AUTEUR}

RICHARD C. TREXLER

Richard C. TREXLER est professeur à la State University of New York à Binghamton. 